\title{
Risk Probability of Having Chronic Kidney Disease over the Past Ten Years Using GH-Method: Math-Physical Medicine
} \begin{abstract}
Haven Way, Woodside, CA 94062 USA.
\section{INTRODUCTION}

The author, who has type 2 diabetes (T2D) for over 25 years, is a research scientist on chronic diseases. He has endured many complications from T2D involving kidney, bladder, foot ulcer, retinopathy, hyperthyroidism, and five cardiac episodes. In this paper, he focuses on investigating the risk probability (Risk) of having chronic kidney disease (CKD).
\end{abstract}

Gerald C. Hsu*

Medical Research Scientist, EclaireMD Fondation, 7 Oak Haven Way, Woodside, CA 94062 USA.

*Corresponding Author: Gerald C. Hsu, Medical Research Scientist, EclaireMD Fondation, 7 Oak

\section{METHODS}

\section{Background:}

The author is a 73-year-old male who has a history of three severe chronic diseases including diabetes, hypertension, and hyperlipidemia for over 25 years. He experienced five cardiac episodes, without having a stroke, from 1994 through 2008. During that period, he thought the cause of his cardiac episodes were outcomes from his stressful business life. In mid-2010, he was diagnosed with an acute renal problem with an albumin-to-creatinine ratio (ACR) level of $116.4 \mathrm{mg} / \mathrm{g}$ (30 -300 mg/g refers to albumin excretion above the normal range) and would eventually require dialysis treatment. At that time, he also consulted with three independent physicians and received similar warnings that he would die within 3 to 5 years if he did not reverse his overall poor health conditions. This kidney episode was his final "wake-up call". Therefore, he launched his own efforts to study and research internal medicine, especially endocrinology. Since 2010 , he spent 30,000 hours to focus on diabetes and its various complications, especially onthe heart and kidney. Now, he has extended his research into diabetic retinopathy and hyperthyroidism.

During 2010-2013, he self-studied diabetes and food nutrition via reading many textbooks and medical publications. In 2012, he developed a customized computer software to collect vital data of his own health conditions. By 2014, he further developed a complex mathematical model of metabolism and also started his stringent lifestyle management program. As a result, his overall health conditions have been noticeably improving since 2014. In the same year, he decided to gradually reduce the dosage of his three different diabetes medications. By December 8, 2015, he completely stopped taking all kinds of diabetes medications. Within 2015-2016, he also created four mathematical prediction models for body weight, fasting plasma glucose (FPG), postprandial plasma glucose (PPG), and HbA1C values, and achieved accuracy rates above $97 \%$. Due to his high-tech background, he also applied optical physics and artificial intelligence to develop an iPhone APP that can estimate the carbs/sugar amount in his food based on photos, and then predict his finger-piercing PPG level at two hours after the first bite of his meal. For the entire post-medication period of 12/8/2015 through $5 / 5 / 2020$, his lab-tested $\mathrm{HbA} 1 \mathrm{C}$ average value is around $6.6 \%$ which decreased from his peak value of $10 \%$ in 2010 .

\section{Glucose}

Blood vessels such as the arteries and microvessels in our body carry glucose (nutrition) and oxygen via blood flow to our heart and other organs which then provide energy (i.e. metabolism) to live our life. This situation is remarkably similar to the tubes that transmit gasoline and air to the engine and other parts of the car which then provide energy to move the vehicle.

In his various research subjects of diabetes and complications, including heart, stroke, kidney, bladder, nervous system, foot, eyes, and thyroid, he has identified high glucose (diabetes) as the "principal criminal" while high blood pressure (hypertension) and high lipid (hyperlipidemia) are "accessory criminals" that work together to 
Risk Probability of Having Chronic Kidney Disease over the Past Ten Years Using GH-Method: MathPhysical Medicine

cause various diabetes complications. However, for CKD, both glucose and blood pressures serve as "joint criminals" to damage the kidney.

There are many contributing factors to high glucose including two main biological factors which are pancreatic beta cell damage and being overweight (obesity). In total, there are 24 influential factors, such as food, exercise, weather temperature, sleep, stress, and more. Among those many influential factors, the two most prominentare carbs/sugar intake (39\%) and post-meal exercise $(41 \%)$. Not only does exercise reduce inflammation in the organs but also highly effective in decreasing glucose level quickly; therefore, the "post-meal" exercise impacts PPG more obviously. Although some human psychological behaviors, such as overeating and being sedentary, are quite common for most people, diet control is indeed far more complicated than exercising in terms of scope of knowledge, variety of choices, and degree of difficulty.

Once diet and exercise are in place, most likely, glucose and HbA1C will be well controlled. Having glucose control combined with the management of both blood pressure (reduce salt consumption, avoid stress, exercise) and lipid (avoid fat, reduce cholesterol consumption, exercise), the blood system (artery and micro-vessels) will then be in a healthy state. This will definitely reduce the risks of having vascular disease such as CVD andstroke along with micro-vessels and nervous system problems, such as CKD or renal failure, bladder infection, foot ulcer, diabetic retinopathy, and erectile dysfunction, and so forth.

\section{CKD Risk Assessment Model}

Instead of using traditional biology and chemistry, the author utilized mathematics, physics, engineering modeling, and computer science to conduct his research.

In 2018, he developed a sophisticated and complex mathematical model to calculate his risk probability of having CKD. He has spent 30,000 hours to research, collect and process $\sim 2$ million data during the period of 2010-2020.

At first, he built a baseline model, including genetic (steady state and unchangeable conditions), semi-permanent factors (weight, waistline), and bad habits, such as hard to change conditions, such as smoking, alcohol drinking, illicit drugs. Next, he developed a risk probability calculation model of estimating the leakage of micro-blood vessels due to different medical conditions mainly diabetes and hypertension.

Finally, he applied his collected $\sim 100,000$ data of medical conditions regarding chronic diseases and $\sim 1$ million data of lifestyle details, from the past ten years, to calculate their combined contribution to kidney complications, including glucose, blood pressure, kidney, glomeruli, bladder, urinary tract, etc.

For the purpose of this analysis, he made his last but most important step of the calculation. He used lab-tested data of albumin, creatinine, and albumin-creatinine ratio (ACR) from the past ten years as the yardstick of comparison against his input data.

It should be noted here that the risk probability percentages are expressed on a "relative" scale, not on an "absolute" scale.

\section{RESULTS}

Albuminuria categories in $\mathrm{CKD}$, from the American Kidney Association, are reflected in Figure 1. As shown in Figures 2, in 2010, he had stage A2 of CKD (ACR between 30 to 300 $\mathrm{mg} / \mathrm{mmol}$ for greater than 3 months), and this stage may have extended into 2011 and 2012. From 2013 through 2019, his ACR values were below $30 \mathrm{mg} / \mathrm{mmol}$ which indicated that his kidney conditions were back to the Stage A1(normal).

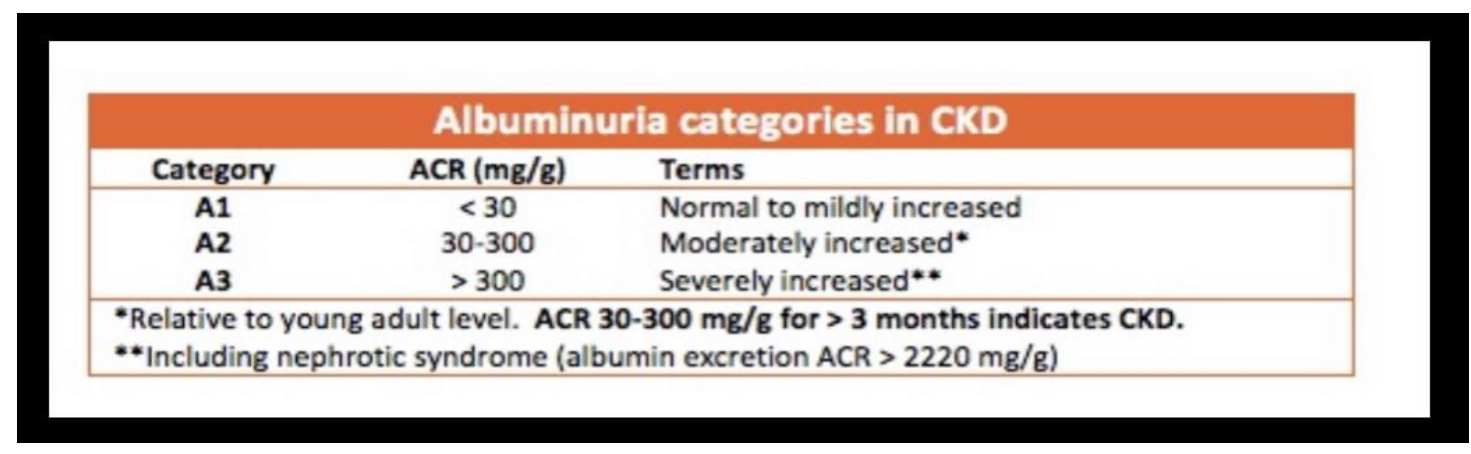

Figure 1: AKA's Albuminuria Categories in CKD 


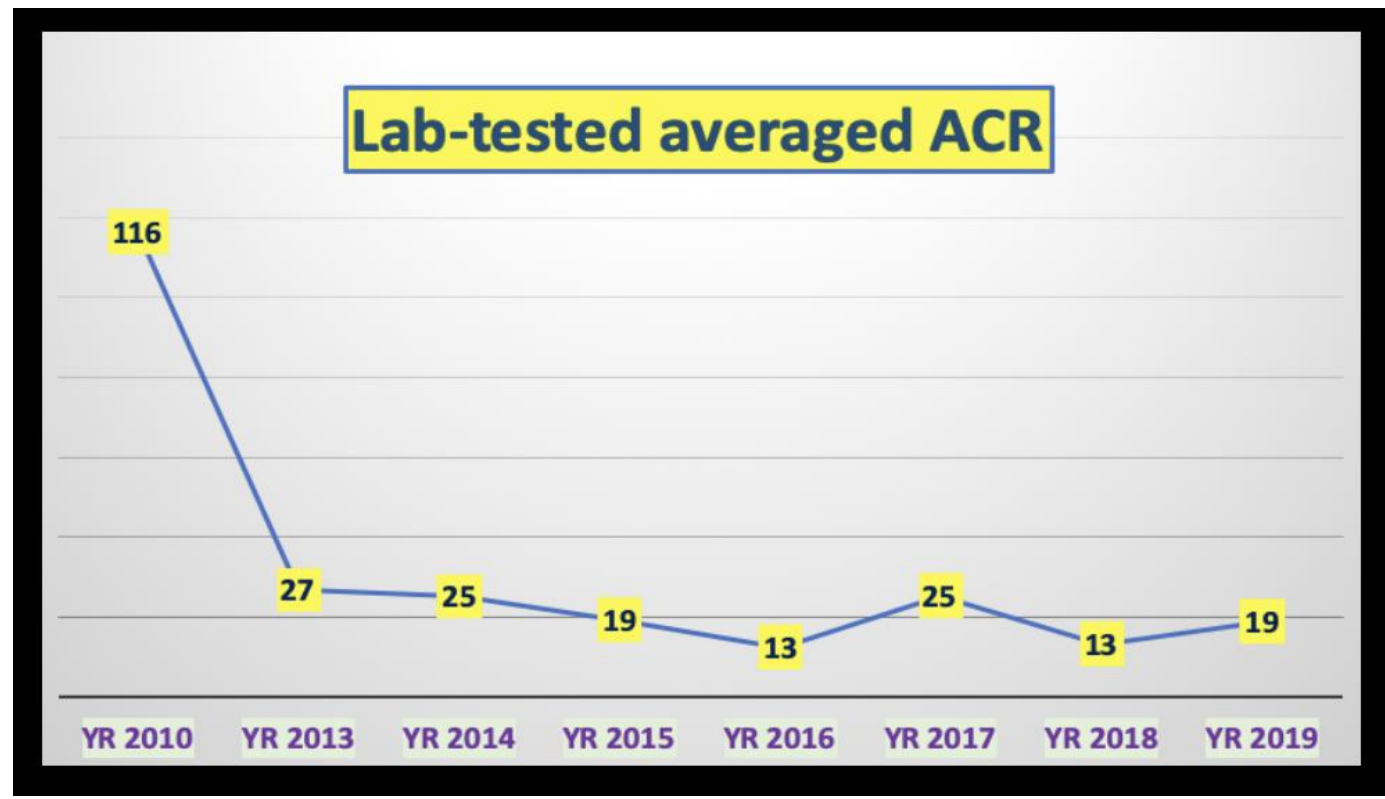

Figure 2: ACR Data (2010-2019)

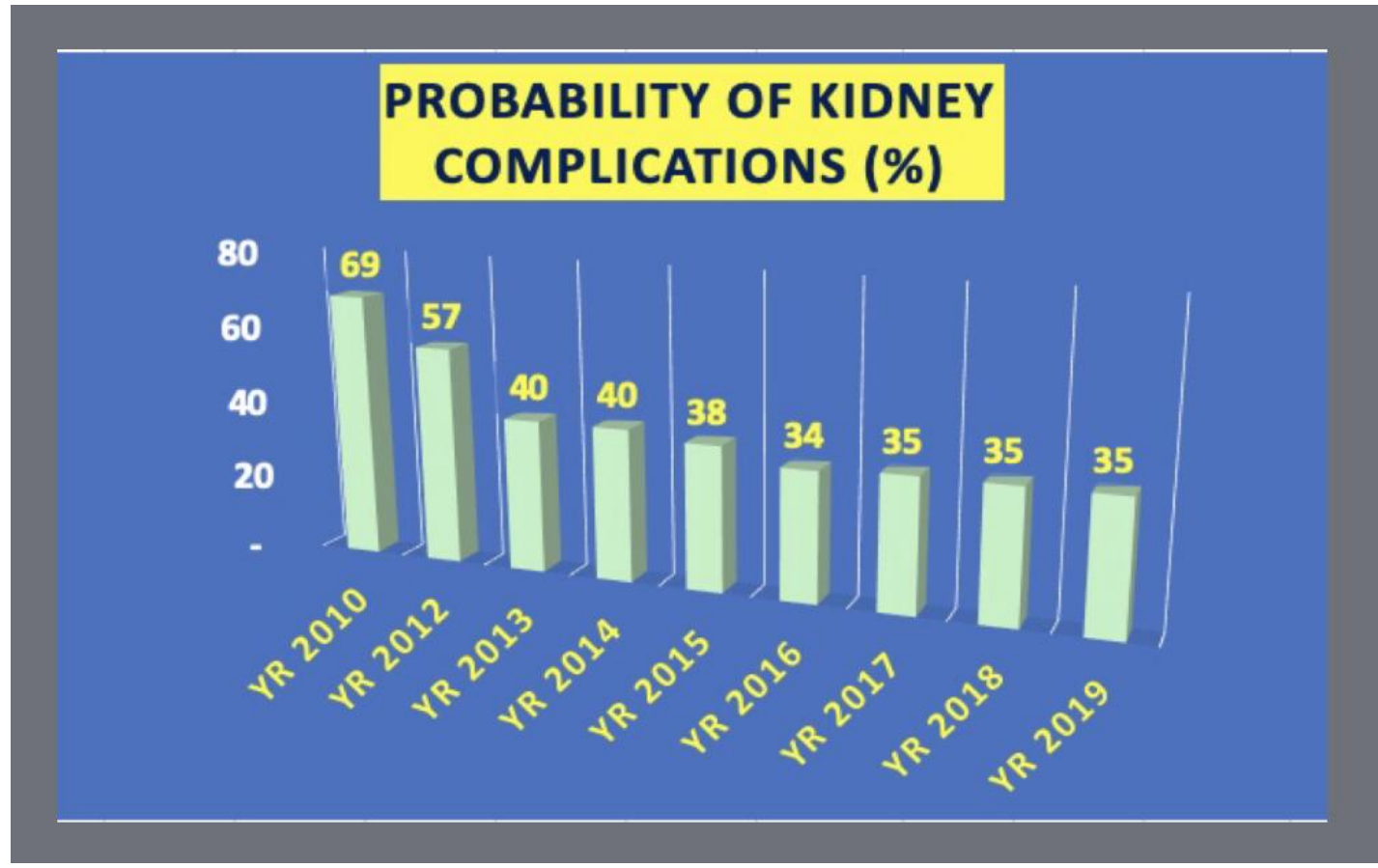

Figure 3: Risk Probability \% of having Renal Complications (CKD)

A bar chart reflects his annual risk probability $\%$ of having a CKD in Figure 3.

Here is his annualized CKD risk probabilities during 2010-2019.

Y2000: (weight 220 lbs., BMI 32.5, and waistline 44 inches)

Y2010: 69\% (weight 198 lbs., BMI 29.2, glucose $280 \mathrm{mg} / \mathrm{dL}$, A1C 10.0,ACR 116.4)

Y2012: 57\% (glucose $128 \mathrm{mg} / \mathrm{dL}$, A1C 6.9)

Y2013: 40\% (glucose $133 \mathrm{mg} / \mathrm{dL}$, A1C 6.9, ACR 27)
Y2014: 40\% (glucose $135 \mathrm{mg} / \mathrm{dL}, \mathrm{A} 1 \mathrm{C} 6.8$, ACR 25)

Y2015: 38\% (glucose $129 \mathrm{mg} / \mathrm{dL}$, A1C 6.6, ACR 19)

Y2016: 34\% (glucose $119 \mathrm{mg} / \mathrm{dL}$, A1C 6.6, ACR 13)

Y2017: 35\% (glucose $117 \mathrm{mg} / \mathrm{dL}$, A1C 6.5, ACR 25)

Y2018: 35\% (glucose $116 \mathrm{mg} / \mathrm{dL}$, A1C 6.8, ACR 13)

Y2019: 35\% (glucose 114mg/dL, A1C 6.7, ACR 19) 
Risk Probability of Having Chronic Kidney Disease over the Past Ten Years Using GH-Method: MathPhysical Medicine

His risks from 2017 thorough 2019 were $35 \%$ which is almost half of his risk level of $69 \%$ during 2010.

The more interesting findings of the triangular relationships among $\mathrm{CKD}$ risk, $\mathrm{ACR}$, and $\mathrm{HbA} 1 \mathrm{C}$ are presented in Figure 4. Those three sets of extremely high correlation coefficients (R) are listed below:

(1) ACR vs. HbA1C: R = 98.7\%

(2) Risk \% vs. ACR: R =97.2\%

(3) RISK \% vs. HbA1C: R = 96.1\%

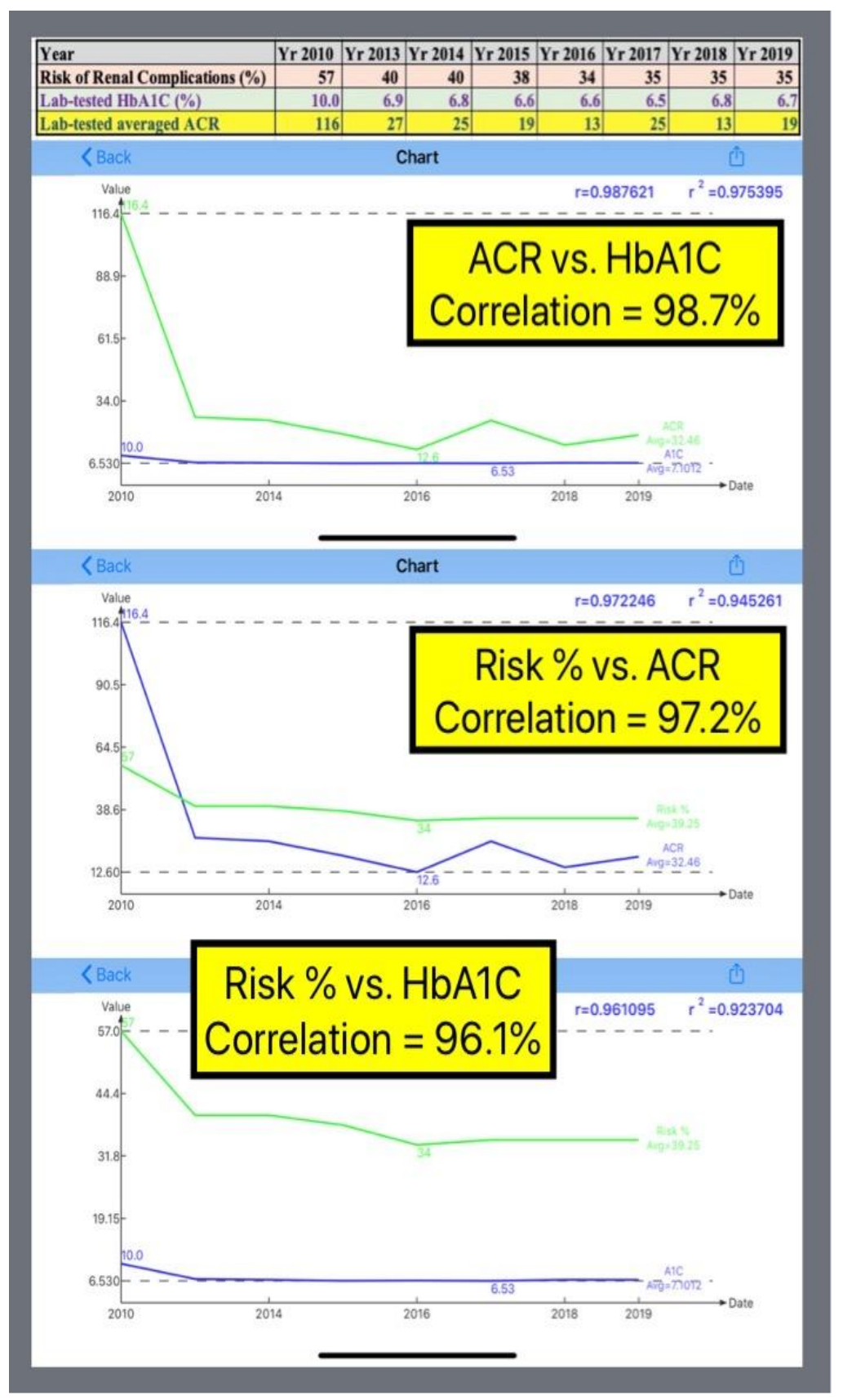

Figure 4: Triangular Correlations among CKD Risk, ACR, \& HbAlC

The above numerical results of extremely high correlation coefficients, greater than $96 \%$, have demonstrated the strong relationship among CKD risk, $\mathrm{ACR}$, and $\mathrm{HbA} 1 \mathrm{C}$.

\section{CONCLUSION}

This investigation does not focus on kidney data (ACR) alone. His main purpose is to study the relationship between kidney complications and chronic diseases, especially T2D and CKD, from a larger pool of associated data. In addition, it has proven that glucose having the role as the "principal criminal" along with the help of blood pressure as the "accessory criminal" committed together to cause kidney damage. 
Risk Probability of Having Chronic Kidney Disease over the Past Ten Years Using GH-Method: MathPhysical Medicine

\section{REFERENCES}

[1] Hsu, Gerald C., eclaireMD Foundation, USA, No. 34 "Risk Probability of Kidney Complications Resulting from Chronic Diseases (Math-Physical Medicine)"

[2] Hsu, Gerald C., eclaireMD Foundation, USA, No. 39 "Using GH-Method: Math-Physical Medicine to Investigate the Risk Probability of Renal Complications from Chronic Diseases with Input from Sensor-Based Glucose Data"

[3] Hsu, Gerald C., eclaireMD Foundation, USA, No. 64 "Using GH-Method: math-physical medicine and segmented glucose pattern analysis to investigate the variance of risk probability of having renal complications"

[4] Hsu, Gerald C., eclaireMD Foundation, USA, No. 117 "Using GH-Method: math-physical medicine to investigate the risk probability of metabolic disorders induced cardiovascular diseases, stroke, and renal complications"

[5] Hsu, Gerald C., eclaireMD Foundation, USA, No. 142 "Risk probability of having a renal complication using annual segmented data of glucose and metabolism index (GH Method: math-physical medicine)“

Citation: Gerald C. Hsu, "Risk Probability of Having Chronic Kidney Disease over the Past Ten Years Using GH-Method: Math-Physical Medicine”, International Journal of Research Studies in Medical and Health Sciences. 2020; 5(6): 07-11.

Copyright: (c) 2020 Gerald C. Hsu, This is an open-access article distributed under the terms of the Creative Commons Attribution License, which permits unrestricted use, distribution, and reproduction in any medium, provided the original author and source are credited. 